\title{
Age at menarche as a predictor of diminished ovarian reserve: a new approach
}

\author{
Ramona Perhar, Rita Shukla, Akanksha Mishra*
}

Department of Obstetrics \& Gynaecology, M.L.N Medical College, Allahabad, Uttar Pradesh, India

Received: 28 December 2014

Accepted: 11 January 2015

\author{
*Correspondence: \\ Dr. Akanksha Mishra, \\ E-mail: dr.akanksha.gsvm@gmail.com
}

Copyright: (C) the author(s), publisher and licensee Medip Academy. This is an open-access article distributed under the terms of the Creative Commons Attribution Non-Commercial License, which permits unrestricted non-commercial use, distribution, and reproduction in any medium, provided the original work is properly cited.

\begin{abstract}
Background: Objective of current study was to investigate whether age at menarche is associated with Functional Ovarian Reserve (FOR) later in life.

Methods: Design: Retrospective cohort study. Setting: Gayatri Hospital, Allahabad, Uttar Pradesh, India. Patient(s): One hundred fifty one. Intervention(s): None.

Results: The mean age of the patients was $38.9 \pm 4.9$ years, and their mean level of AMH was $1.4 \pm 2.0 \mathrm{ng} / \mathrm{ml} \mathrm{and}$ of FSH was $10.7 \pm 6.1 \mathrm{mIU} / \mathrm{ml}$. Their current age-specific Diminished Functional Ovarian Reserve (DFOR) was statistically significantly associated with early menarche, defined as age $<13$ years. Logistic regression analysis affirmed the higher likelihood of early age of menarche in infertile patients with DFOR. When women with DFOR were grouped into quartiles, early menarche $\left(<25^{\text {th }}\right.$ percentile) was associated with statistically significantly higher DFOR risk than late menarche $\left(>75^{\text {th }}\right.$ percentile).

Conclusions: The study demonstrates a statistically significant impact of menarche on DFOR risk later in life among infertile women. The occurrence of menarche may relate to follicular pool size and/or speed of follicle recruitment, which in turn, is predictive of occurrence of DFOR later in life.
\end{abstract}

Keywords: Anti-Mullerian hormone (AMH), follicle-stimulating hormone (FSH), Diminished functional ovarian reserve, menarche, Ovarian function

\section{INTRODUCTION}

Women in the industrialized world increasingly defer family building. ${ }^{1,2}$ As fecundity declines with age, ${ }^{3}$ the ability to prospectively estimate remaining reproductive life span increases in importance. Prospectively determining a woman's risk of developing Diminished Functional Ovarian Reserve (DFOR), and thus reduced pregnancy chances during older age would thus be helpful.

Because the currently used ovarian reserve markers are mainly tailored to assessing present Functional Ovarian Reserve (FOR), ${ }^{4}$ they may fail to predict a woman's ovarian aging curve at later stages of life. ${ }^{5}$ Anti Mullerian hormone levels also have recently been suggested to be predictive of menopause. ${ }^{6}$ AntiMullerian hormone levels have lately been suggested to be predictive of menopause. $^{6}$

Menopause, one stage in ovarian aging, convincingly reflects a woman's follicle pool. At that point, only a small number of follicles are left in ovaries, generally considered below $1000 .^{7,8}$ Menopause appears to be genetically preprogrammed. Schuh Huerta et al. ${ }^{9}$ demonstrated shared genetic loci for follicular pool size and menopausal age. He et al. ${ }^{10}$ reached similar conclusions when noting that gene clusters of steroid hormone synthesis and metabolism were involved in premature ovarian failure and physiological age at menopause. Genetic control of menopause is also suggested by high correlations of menopausal age between mothers and daughters. ${ }^{11}$ 
Comparable associations have also been reported for age at menarche. Twin studies suggest significant concordance of age at menarche Twin studies suggest significant concordance of age at menarche between monozygotic and dizygotic twins. ${ }^{12}$ Generational studies, in turn, have suggested that ethnicity and mother's and grandmothers ages at menarche are highly predictive parameters for menarchal age(13).

Though timing of menarche cannot be attributed to a single trigger, reactivation of gonadotropin-releasing hormone $(\mathrm{GnRH})$ pulsatility has been assumed essential. ${ }^{14}$ The hypothesis that GnRH is influenced by ovarian activity, and the observation that follicular recruitment peaks around menarche suggest a possible relationship between menarchal age and a woman's follicular pool. ${ }^{14,15}$

\section{METHODS}

This study investigated 251 consecutive infertility patients who underwent fertility evaluations at Gayatri hospital in Allahabad city of Uttar Pradesh, India between January 2007 and June 2013.

All patients had a routine baseline evaluation; in addition to a detailed medical history, including age, Body Mass Index (BMI) age at menarche, and indication for fertility treatments, they also received a baseline laboratory evaluation of ovarian function parameters. The latter included measurement of day 2-3 follicle-stimulating hormone (FSH) and estradiol levels, and measurement of $\mathrm{AMH}$ on random cycle days. All relevant deidentified patient data were then entered into an electronic research database.

A diagnosis of DFOR was established, as previously reported, if a woman presented with an abnormally low age-specific antiMullerian hormone (AMH) level. These levels have been established as AMH levels below $25^{\text {th }}$ percentile at each 5-year age group, as previously reported in detail elsewhere.

To assess the impact of age at menarche on later ovarian function parameters, the age at menarche was categorized as early or late based on a median age at menarche at 13.0 years: early $<13$ years and late $\geq 13$ years. The DOFR risk was then compared between women with early and late occurrence of menarche. Additionally, the study group was categorized into menarchal age quartiles to compare the DFOR risk between the lowest and the highest quartiles.

The data we obtained only involved retrospective review of medical records and deidentified research database. Patients at our centre signed an informed consent at initial consultation, which allows for such reviews if the patients' medical record remains confidential and her identity remains protected.

\section{Statistical analysis}

Non parametric tests - namely, the Mann-Whitney U test and chi-square tests - were performed to assess the potentially confounding factors of age-specific ovarian function like BMI. Logistic regression was then used to examine the impact of age at menarche on ovarian function, categorized as the presence or lack of DFOR. The baseline characteristics of patients with early and late menarche were compared using t-tests.

Continuous data are presented as mean \pm standard deviation (SD). All tests were two-tailed and $\mathrm{P}<0.05$ was considered statistically significant. In logistic regression models, alpha was set at 0.05 with regards to the individual contributors of predictor variables.

\section{RESULTS}

Table 1 summarizes the patient characteristics. The mean age for investigated patients was $38.9 \pm 4.9$ years.

\section{Table 1: Patient characteristics of 251 infertile women} undergoingfertility evaluation.

\begin{tabular}{|lll|}
\hline & Mean \pm SD & Median (IQR) \\
\hline Female age (y) & $38.9 \pm 4.9$ & $39.7(35.6,42.6)$ \\
\hline AMH (ng/ml) & $1.4 \pm 2.0$ & $23.0(20.8,26.6)$ \\
\hline Baseline FSH (mIU/MI) & $10.7 \pm 6.1$ & $0.8(0.2,1.8)$ \\
\hline $\begin{array}{l}\text { Baseline estradiol } \\
\text { (pg/ml) }\end{array}$ & $52.2 \pm 27.2$ & $9.3(7.0,12.6)$ \\
\hline BMI (kg/sq.m) & $24.4 \pm 5.0$ & $47.7(34.0,63.4)$ \\
\hline Age at menarche (y) & $12.9 \pm 1.7$ & $13.0(12.0,14.0)$ \\
\hline Prevalence of DOFR by age (y) & \\
\hline$<35$ & 22 & 42.6 \\
\hline $35-37$ & 15 & 53.7 \\
\hline $38-40$ & 33 & 61.3 \\
\hline $41-42$ & 33 & 58.0 \\
\hline$>42$ & 39 & 59.7 \\
\hline Prevalence of DOFR by age at menarche (y) \\
\hline$<12$ & 61 & 62.7 \\
\hline $12-13$ & 39 & 50.7 \\
\hline$\geq 14$ & 40 & 51.7 \\
\hline
\end{tabular}

Indications for fertility treatment were tubal factor in $16.7 \%$, diminished ovarian reserve in $62.5 \%$, polycystic ovarian syndrome (PCOS) in $7.2 \%$, male factor infertility in $20.7 \%$, and others in $5.2 \%$ (multiple entries possible); $95.4 \%$ of all participants had a history of female factor infertility.

A statistically significant association between menarcheal age and DFOR, reflected in abnormal age-specific ovarian function testing, was observed. We found DFOR in $61(62.7 \%)$ of 97 women with early menarche compared with $78(51.1 \%$ ) of 154 with late menarche (chi square $[(1, \mathrm{n}=251)=6.43 ; \mathrm{P}=0.01$, OR 1.6; confidence interval (CI), 1.1, 2.3]. 
When women with DOFR were grouped into quartiles: $43.4 \%$ entered menarche at age $\leq 12.0$ years $\left(25^{\text {th }}\right.$ percentile), $27.6 \%$ entered menarche between the ages of 12.1 and 13.4 years (interquartile range), and 29.0\% entered menarche at a later age $\left(>75^{\text {th }}\right.$ percentile). Women with early menarche $\left(<25^{\text {th }}\right.$ percentile $)$ were more likely to have DFOR than the women who had experienced menarche at a later age $\left(>75^{\text {th }}\right.$ percentile) (OR 1.6; $95 \mathrm{CI}, 1.03,2.42 ; \mathrm{P}=0.04)$. The results also demonstrate an increased DFOR risk in women who undergo menarche earlier $\left(<25^{\text {th }}\right.$ percentile) than the women falling into the interquartile range of 12.1-13.4 years at menarche (OR 1.6; $95 \% \mathrm{CI}, 1.1,2.5 ; \mathrm{P}=0.03)$. Women entering menarche at a later age $\left(<75^{\text {th }}\right.$ percentile) had similar odds of having DFOR as the women entering menarche in the interquartile range (OR $1.04 ; 95 \% \mathrm{CI}, 0.6 ; \mathrm{P}=0.87$ ).

\section{DISCUSSION}

Our results with infertile women have demonstrated a statistically significant association between menarcheal age and DFOR later in life. Women who experienced menarche at a younger age were more likely to suffer from abnormally age-specific DFOR. These results were confirmed when the study group was divided into quartiles, pointing toward a relationship between follicular depletion, even already at very young ages, and reproductive life span.

Though the recently described likely presence of ovarian stem cells raises questions about the current concepts of ovarian senescence, ${ }^{18}$ a good number of studies in women with compromised follicle pools support a correlation between follicle pool size and reproductive lifespan. ${ }^{19-22}$ A retrospective analysis of risk factors for primary ovarian insufficiency/POF in 24152 Japanese women demonstrated statistically significant associations between later menarche and a reduced risk of early menopause and POF.

To solely attribute ovarian function to follicle pool size, however, may be limiting, although our findings on increased DFOR risk in women with earlier menarche as well as generation analyses and genomewide association studies may support such an assumption. The current literature, indeed, convincingly demonstrates that a woman's reproductive life span to a large extent is genetically predetermined. ${ }^{9,13}$ Hefler et al., ${ }^{23}$ for instance, show a statistically significant association between various polymorphisms and age at menopause. In contrast, data by Otero et al. ${ }^{24}$ lack correlations between age at menarche and age at menopause.

Our findings of a statistically significant association between age at menarche and subsequent DFOR risk later in life also suggest the question of whether such an association is attributable to ovarian follicle pool size, follicular depletion rate, or possibly a combination of both. Wallace and Kelsey, ${ }^{8}$ for instance, elegantly present mathematical models that support the assumption that women with large follicle pools also demonstrate more extensive monthly follicle recruitment. They, however, also suggest a positive correlation between follicle pool size and menopausal age. ${ }^{8}$ If, indeed, we assume an association among follicle pool size, follicular recruitment pace, age at menarche, and DFOR, we may also speculate that more extensive follicular recruitment leads to rapid follicular depletion and higher DFOR risk at middle ages- despite a large initial ovarian follicle reserve.

Some POF studies have further supported the assumption that recruitment pace may be essential for the risk of diminished ovarian function: if follicle pool sizes and depletion rates are genetically influenced, as considerable scientific evidence suggests, ${ }^{9}$ higher POF rates after unilateral oophorectomy ${ }^{20}$ likely reflect that depletion rates are programmed and not adjusted under conditions of non-physiologic changes in follicle pool size. Flemming et al. ${ }^{15}$ go even further by demonstrating that follicular recruitment rates change in an age dependent fashion: in healthy girls, follicular recruitment peaks around menarche and declines thereafter throughout the reproductive years. Consequently, AMH should experience peak levels when menarche is reached. AMH levels, and fertility potential are highly correlated. ${ }^{15}$ These findings support our data on the predictive capacity of age at menarche for subsequent DFOR risk during later life.

The retrospective design of our study has to be seen as a limitation, though prospective evaluation of this hypothesis would require long-term observations and would thus appear to be difficult to perform. Additionally, a small minority of women (4.6\%) underwent fertility evaluation due to male factor infertility. Compared with the study participants with female factor related infertility, this subgroup of patients presented with a comparable mean age at menarche; when excluded from the analysis, the differences observed in DFOR risk remain unchanged. However, this may be a limitation of the present study.

Our results suggest that the odds of developing DFOR at a more advanced age are increased for women who had an early rather than a late age at menarche. Because this study was performed in infertile women, the data presented are only applicable to infertile women, but it appears likely that our outlined model of ovarian aging may be generally applicable.

\section{Funding: No funding sources \\ Conflict of interest: None declared \\ Ethical approval: The study was approved by the institutional ethics committee}

\section{REFERENCES}

1. Martin JA, Hamilton BE, Ventura SJ, Osterman MJ, Kirmeyer S, Mathews TJ, et al. Births: final data 2009. Natl Vital Stat Rep. 2011;60:1-70. 
2. Blondel B, Lelong N, Kermarrec M, Goffinet F. Trends in perinatal health in France from 1995 to 2010. Results from the French National Perinatal Surveys. J Gynaecol Obstet Biol Reprod (Paris). 2012;41:e1-15.

3. Nikolaou D, Templeton A. Early ovarian ageing. Eur J Obstet Gynaecol Reprod Biol. 2004;113:126-33.

4. Gleicher N, Weghofer A, Barad DH. Defining ovarian reserve to better understand ovarian aging. Reprod Biol Endocrinol. 2011;9:23.

5. Loh JS, Maheshwari A. Anti-Mullerian hormone: it is a crystal ball for predicting ovarian ageing? Hum Reprod. 2011;26:2925-32.

6. Broer SL, Eijkemans MJ, Scheffer GJ, van Rooji IA, de Vet A, Themmem AP, et al. Anti-Mullerian hormone predicts menopause: a long term follow-up study in normoovulatory women. J Clin Endocrinol Metab. 2011;96:2532-9.

7. Broekmans FJ, Soules MR, Fauser BC. Ovarian aging: mechanisms and clinical consequences. Endocr Rev. 2009;30:465-93.

8. Wallace WH, Kelsey TW. Human ovarian reserve from conception to menopause. PLoS One. 2010;5:e8772.

9. Schuh-Huerta SM, Jhonson NA, Rosen MP, Sternfeld B, Cedars MI, Reijo Pera RA. Genetic markers of ovarian follicle number and menopause in women of multiple ethnicities. Hum Genet. 2012;131:1709-24.

10. He C, Kraft P, Chasman DI, Buring JE, Chen C, Hankinson SE, et al. A large scale candidate gene association study of age at menarche and age at natural menopause in women of multiple ethnicities. Hum Genet. 2010;128:515-27.

11. Murray A, Bennett CE, Perry JR, Weedon MN, Jacobs PA, Morris DH, et al. Common genetic variants are significant risk factors for early menopause: Results from the Breakthrough Generations Study. Hum Mol Genet. 2010;20:18693.

12. Fischbein S. Onset of puberty in $\mathrm{MX}$ and $\mathrm{DZ}$ twins. Acta Med Gemellol (Roma). 1997;26:151-8.

13. Chang SR, Chen KH. Age at menarche of threegeneration families in Taiwan. Ann Hum Biol. 2008;8:115.

14. Karapanou O, Papadimitriou A. Determinants of menarche. Reprod Biol Endocrinol. 2010;8:115.
15. Fleming R, Kelsey TW, Anderson RA, Wallace WH, Nelson SM. Interpreting human follicular and antiMullerian hormone concentrations throughout life. Fertil Steril. 2012;98:1097-102.

16. Santoro N. Mechanisms of premature ovarian failure. Ann Endocrinol (Paris). 2003;64:87-92.

17. Barad DH, Wehhofer A, Gleicher N. Utility of agespecific serum anti-Mullerian hormone concentrations. Reprod Biomed Online. 2011;22:284-91.

18. White YA, Woods DC, Takai Y, Ishihara O, Seki H, Tilly JL. Oocyte formation by mitotically active germ cells purified from ovaries of reproductive-age women. Nat Med. 2012;18:413-21.

19. Chang SH, Kim CS, Lee KS, Kim H, Yim SV, Lim YJ, et al. Premenopausal factors influencing premature ovarian failure and early menopause. Maturitas. 2007;58:19-30.

20. Yasui T, Hayashi K, Mizunuma H, Kubota T, Aso T, Matsumura Y, et al. Factors associated with premature ovarian failure, early menopause in Japanese women. Maturitas. 2012;72:249-55.

21. Hovatta O. Ovarian function and in vitro fertilization (IVF) in Turner syndrome. Paediatr Endocrinol Rev. 2012;9(Suppl 2):713-7.

22. Sadrzadeh S, Klip WA, Broekmans SJ, Schats R, Willemsen WN, Berger CW, et al. Birth weight and age at menarche of patients with polycystic ovary syndrome or diminished ovarian reserve, in a retrospective cohort. Hum Reprod. 2003;18:2225-30.

23. Hefler LA, Grimm C, Bentz EK, Reinthallar A, Heinze G, Tempfer CB. A model for predicting age at menopause in white women. Fertil Steril. 2006;85:451-4.

24. Otero UB, Chor D, Carvalho MS, Faerstein E, Lopes $\mathrm{C}$ de S, Werneck GL. Lack of association between age at menarche and age at menopause: Pro-Saude Study Rio de Janeiro, Brazil. Maturitas. 2010;67:245-50.

DOI: $10.5455 / 2320-1770 . i j r \operatorname{cog} 20150242$

Cite this article as: Perhar R, Shukla R, Mishra A. Age at menarche as a predictor of diminished ovarian reserve: a new approach. Int J Reprod Contracept Obstet Gynecol 2015;4:235-8. 